\title{
A Water-Cooled Mirror System for Synchrotron Radiation
}

LBL -23777

Richard DiGennaro, Bruce Gee, Jim Guigli,

DE88 000206

Henning Hogrefe, and Malcolm Howells

Center for X-Ray Optics, Lawrence Berkeley Laboratory

University of Califomia, Berkeley, Califorria 94720

Harvey Rarback

National Synchrotron Light Source,

Brookhaven National Laboratory

Upton, New York 11973

Presented at the 5 th National Conference on

Synchrotron Radiation Instrumentation

June 21-25, 1987

Madison, Wisconsin

Contact:: Richard DiGennaro

Lawrence Berkeley Laboratory

University of California

\#1 Cyclotron Road, Mail Stop 46-161

Berkeley, California 94720

Phone: (415) 486-6466

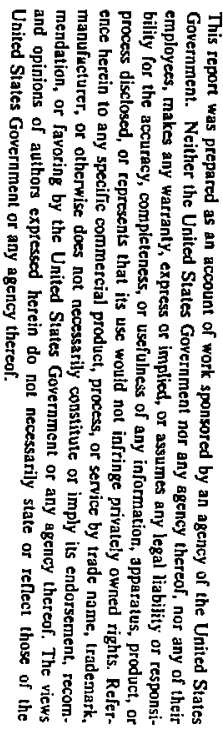




\title{
A Water-Cooled Mirror System for Synchrotron Radiation
}

\author{
Richard DiGennaro, Brice Gee, Jim Guigli, \\ Henning Hogrefe, and Malcolm Howells \\ Center for X-Ray Optics, Lawrence Berkeley Laboratory \\ University of California, Berkeley, California 94720 \\ Harvey Rarback \\ National Synchrotron Light Source, \\ Brookhaven National Laboratory, Upton, New York 11973
}

\begin{abstract}
This paper describes the design and performance of a directly-cooled soft $x$-ray mirror system which has been developed at Lawrence Berkeley Laboratory for synchrotron radiation beam lines in which mirror thermal distoition must be minimized for acceptable optical performance. ${ }^{1}$ Two similar mirror systems are being built: the first mirror has been installed and operated at the National Synchrotron Light Sousce on the X-17T mini-undulator beam line and will be moved to the permanent $X-1$ beam line when a new, more powerful undulator is installed there. The second system is being built for installation at the Stanford Synchrotron Radiation Laboratory on Beam Line VI, where the total absorbed power on the mirror may be as high as $2400 \mathrm{~W}$ with peak absorbed power density of $520 \mathrm{~W} / \mathrm{cm}^{2}$. Direct cooling by convection is achieved using internal water channels in a brazed, dispersion-strengthened copper and OFHC copper substrate with a polished electroless-nickel surface. A simple kinematic linkage and flexural pivot mounting provide for mirror positioning about two rotational axes that coincide with the optical surface. Surface figure metrology, optical configurations, and tolerancing are also discussed.
\end{abstract}

1 This work was supported by the Office of Basic Energy Sciences, U. S. Department of Energy, under conlract \# DE-ACO3-76SF00098. 


\section{Introduction}

Thernal design issues for synchrotron radiation instrumentation continue to be among the primary factors that strongly influence bean line performance and cost. Thermal loading, combined with additional requirements for ultra-high vacuum (UHV), safety, reliability, stability requirements and optical system precision, consumes significant engineering effort in order to assure acceptable performance. [1]

Also, in order to exploit low emittance characteristics of modem synchrotron sources, beam line optical system designers are required to achieve high spatial and wavelength resolution. Thermal distortion of optical surfaces in high flux beam lines must therefore be minimized in order not to linit the imaging quality of the system.

Lawrence Berkeley Laboratory (LBL) has developed a directly-cooled soft $\mathrm{x}$-ray mirror system for synchrotron radiation beam lines where miror thermal distortion must be minimized for acceptable oprical performance. [2] Two similar mirror systems are being built in which direct mirror cooling by convection is achieved using internal water channels in a brazed, dispersion-strengthened copper alloy [3] and OFHC copper substrate, with a polished electroless-nickel surface. The first mirror has been installed and operated at the National Synchrotron Light Source (NSLS) on the X17 T mini-undulator beam line and will be moved to the permanent $X-1$ beam line when a new, more powerful undulator is installed there. $[4,5]$ The $X-1$ beam line will be used primarily for soft $x$-ray imaging using such methods as microscopy and holography, in the wavelength range of 1 to $5 \mathrm{~nm}$. The total incident power on the mirror may be as high as $720 \mathrm{~W}$ with a peak power density of about $70 \mathrm{~W} / \mathrm{cm}^{2}$.

The second niror is being built for installation at the Stanford Synchrotron Radiation Laboratory (SSRL) on Beam Line VI with the LBL/Exxon/SSRL 54-pole wiggler source, where the total absorbed power on the mirror may be as high as $2400 \mathrm{~W}$ with a peak absorbed power density of $520 \mathrm{~W} / \mathrm{cm}^{2}$. This new branch line for Beam Line VI is intended to provide high photon flux with good spectral resolution in the wavelength range of 1 to $30 \mathrm{~nm}$. 


\section{Optical Configuration and Tolerancing}

In both beam lines, at NSLS and at SSRL, the mirror is used as a simple, high-power beam deflector which separates part of the prinary beam as a branch line from the straight-through beam. The other task performed by the mirror is that of a low-pass photon energy filter to absorb the hard $x$-ray photons which contain most of the beam power. This must be done without distorting the optical wavefront beyond acceptable limits (see below). The beam line optical configurations and important beam line parameters are given in Figures la and $1 \mathrm{~b}$.

These configurations determine the tolerance requirements as well as optical and mechanical layout of the mirror systems. For ideal optical elements along a beam line, the source size (approximately $2 \sigma_{\mathrm{H}} \times 2 \sigma_{\mathrm{V}}$ ) limits the optical perfonmance that can be achieved at the experiment - usually measured in terms of flux, spot size, and energy resolution. Degradation of optimum performance introduced by real, non-ideal optical elements can arise from mechanical and surface errors. Maxinum allowable surface slope errors $\Delta$, i.e. errors that account for long surface wavelengths (geometrical optics regime), can be derived from the requirement that the virtual image of the source in the plane deflection mirror shall not be significantly larger than the real source. This implies:

$$
\begin{array}{ll}
\text { In horizontal (tangential) plane : } & \Delta_{\mathrm{t}}=2 \sigma_{\mathrm{H}} / 2 \mathrm{r} \\
\text { In vertical (sagittal) plane: } & \Delta_{\mathrm{S}}=2 \sigma_{\mathrm{V}} / 2 \mathrm{r} \theta
\end{array}
$$

Actual figure errors should be even a factor of 3 to 10 smaller than $\Delta_{\mathrm{l}}$ and $\Delta_{\mathrm{S}}$ in order to avoid significant image broadening due to miror slope errors.

From the above equations, we can see that mirror performance is much more forgiving to sagiltal slope errors compared with tangential errors - by a factor of $1 / \theta$. This effect is exploited in our schemes by the choice of horizontal deflection. The long narrow beam footprint on the mirror, which results from this choice in grazing incidence, causes figure errors primarily in the sagittal direction and therefore has reduced effect on image broadening. Water cooling is needed to keep both temperatures and thermal stresses low. Because of the smaller source size and the larger object distance, slope tolerances are tighter for the NSLS X-1 beam line than for SSRL Beam Line 
VI. At NSLS, with a source size of $2 \sigma_{\mathrm{H}} \times 2 \sigma_{\mathrm{V}} \cong 1 \times 0.2 \mathrm{~mm}^{2}$ and angle of incidence of $\theta=40$ mrad, we have $\Delta_{\mathrm{l}}=8$ arcsec and $\Delta_{\mathrm{S}}=40$ arcsec. At SSRL, $\Delta_{\mathrm{t}}=100$ arcsec and $\Delta_{\mathrm{S}}=150 \mathrm{arcsec}$, but thermal distortion is also much more severe.

Allowing for thermal distorion and a margin of safety, our specifications for optical fabrication were 1 arcsec rms slope error in all directions for both mirrors, or $\lambda / 20$ over any $5 \mathrm{~mm}$ along the surface. For a plane mirror, this is a fairly generous tolerance in precision optics, the main complications in this case being the heavy, unbalanced metal substrate with internal water-cooling chinnels $3 \mathrm{~mm}$ below the optical surface.

Surface microroughness, which we define heuristically to mean surface imperfections with spatial wavelengths smaller than about $5 \mathrm{~mm}$ (physical optics regime), causes an undesirable stray light background in the optical system. We specified rms surface roughness $\sigma \leq 10 \AA$, a value that sequires advanced polishing techniques for electroless nickel coated metal surfaces but is nontheless regularly achieved by good optical fabricators.

\section{Mirror Thermal Loading}

The mirror on the SSRL beam line will receive up to $2400 \mathrm{~W}$ total absorbed power with peak power density of more than $520 \mathrm{~W} / \mathrm{cm}^{2}$. At this power level, direct water-cooling of the mirror substrate is required. Without direct cooling, substrate temperatures, distortion, and thermal stresses would be unacceptably high; mirror coatings and substrate materials could not survive maximum thermal loads. An optimized geometry for internal water channels in a metal substrate has been chosen, which comprises a single cooling passage matched to the long, narrow beam footprint. With this configuration, calculated maximum thermal stress is less than $1 / 4$ of the measured $0.01 \%$ offset yield strength of $35,000 \mathrm{psi}$ for the substrate material [6], and peak surface temperatures will be less than $60^{\circ} \mathrm{C}$. Thus, the mirror is expected to be able to withstand maximum beam power, corresponding to being plunged into the center of the beam of the wiggler at minimum gap and $200 \mathrm{mAmp}$ storage ring current at $3.0 \mathrm{GeV}$ with no permanent distortion or damage to the optical surface and temporary distortion within the optical limits.

Finite element analysis using a fine-mesh, 2-dinensional model (Figures 2a,3a,3b) for calculating thermal distortion at varying power levels on Beam Line VI indicate that maximum sagittal slope 
error at the optical surface is directly proportional to the peak absorbed power density. At 520 $W / \mathrm{cm}^{2}$, maxinum sagittal slope error due to thermal distortion is expected to be less than 60 arcsec in the illuminated region.

Although the absorbed power is significantly less for the mirror system on the X-1 Beam Line, thermal distortion tolerance is also somewhat lower in order to achieve high photon flux through a collimating pinhole located downstream from the mirror. Here, the maximum sagittal slope error due to thermal distortion is expected to be less than 8 arcsec. Therefore, with direct water-cooling, both nirrors can be kept within the maximum allowable $\Delta_{\mathrm{S}}$ values specified above.

Additional finite element analysis with a 3-dimensional model (Figure 2b) to estimate the tangential slope errors, $\Delta_{\mathfrak{t}}$, due to thermal distortion show that the figure error is primarily due to gross temperature variations within the mirror substrate neur the edges of the illuminated region. On Beam Line VI, the figure error is significant only at the mirror ends where there is an abrupt transition from the illuminated region to an unheated region of water-cooled substrate, causing large temperature gradients there. Only under unusual conditions of maximum thermal loading in which the downstream end of the mirror could receive central beam power, would $\Delta_{t}$ exceed the specified 100 arcsec tolerance in the region within about 1 to $2 \mathrm{~cm}$ from the end. Even then, about $90 \%$ of the mirror length would remain within tolerance for $\Delta_{\mathrm{t}}$. Relatively small tangential figure errors over most of the mirror length are mainly due to gross bending of the substrate caused by thermal expansion along the illuminated surface.

At NSLS, the mirror is preceded by an aperture-defining device that will absorb much of the unwanted beam power in the horizontal (cangential) direction. The nirror generally receives only a central portion of the undulator radiation. Consequently, tangential errors due to thermal distortion will be small mainly because of significantly lower total absorbed power - a small fraction of the $720 \mathrm{~W}$ maximum power output from the undulator. Since only a small region of the mirror is illuminated, the bulk of the mirror substrate will be at a uniform temperature, and substrate bending is not significant. As with the SSRL mirror, tangential figure errors are due mainly to temperature gradients near the edges of the illuminated region, but because total power and power densities are much lower, peak surface temperaures are closer to the bulk temperantre of the substrate and gradients are less significant. Maximum tangential figure error is estimated to be about the same as the sagittal error - less than the allowed 8 aresec even with full beam power incident on the mirror. 


\section{Mirror System Design}

The mirror substrate is a fumace-brazed assembly, fabricated with a dispersion-strengthened copper, UNS Copper Alloy C15715 [3,7], which has a high yield strength due to a fine dispersion of alumina particles in an OFHC copper matrix. The brazed mirror assembly is approximately 26 $\mathrm{cm}$ in length and $9 \mathrm{~cm}$ by $9 \mathrm{~cm}$ in cross-section. The clear aperture of the optical surface is $20 \mathrm{~cm}$ by $2 \mathrm{~cm}$. A single water circuit through machined water channels and brazed tubing supplies cooling water close to the optical surface (see Figures 4 and 5 ) and also to an upstream mask which prevents beam impingement on the end of the mirror.

The mirror back is a box-like, machined piece of OFHC copper, brazed to the front substrate in order to provide an air-jacket around all water connections and vent to atmosphere. The mirror back also isolates the clamping forces for mirror mounting the optical surface.

After brazing, the mirror surface was polished, electroless nickel plated, then polished again. At the one end of the mirror where roundoff from polishing must be minimized, a copper extension piece was attached to the mirror for final machining, plating, and polishing, and then removed for operation. (The mirror is thus a "scraper" mirror.) After polishing, cleaning for UHV was done by a vapor degrease, followed by a series of wipe-and-rinse procedures with alcohol and filtered, deionized water.

Mirror mounting provides independent positioning adjustments during operation for three degrees of freedom: roll and yaw, which are the two motions that steer the bears. and horizontal translation (see Figures 6 - 9). The mirror back is rigidly clamped to flexural pivots that are aligned with the (vertical) $y$-axis at the optical surface for yaw positioning adjustments. The flexural pivots are mounted on a hinged-linkage assembly that has a center of rotation which coincides with the (longitudinal) z-axis at the optical surface for roll positioning. A "joy-stick" and bellows are connected to the mirror back for yaw and roll adjustments, with supply and return water tubing passing through the joy-stick. Internal water connections to the mirror and mask use o-ring fittings, with ethylene propylene diene (EPDM) elastomer o-rings for resistance to radiation damage. [8] 
The vent to atmosphere through the joy-stick also allows a convenient feed-through for leads to thermocouples that are embedded in the mirror substrate. This feature provides a way to directly monitor temperatures close to the illuminated surface during operation (and during bake-out as well).

The entire in-vacuum mounting assembly is supported on a rigid translation tube with a second bellows for (horizontal) $x$-axis positioning. Two pairs of constant-force negator springs are used to reduce loads due to atmospheric pressure against vacuum for horizontal translation.

Linear translation slides and encoders for all three motions are driven by stepper motors for remote optration of the position adjustments. Incremental resolution for roll and yaw motion is determined by the absolute encoder count and the joy-stick length: with 2.5 microns per count and joy-stick length of about $66 \mathrm{~cm}$, angular incremental resolution that can be achieved is 0.8 arcsec over a range of adjustment of about $\pm 2^{\circ}$. The mirror mounting and positioning system is mounted on a single flange and then supported on the vacuum chamber, which is aligned at installation for pitch, elevation, and longitudinal position.

The mirror used at NSLS also takes advantage of the two internal water-cooling channels (for water supply and retum) by using different optical coatings on two separate clear apertures, one at each channel. In order to vary the cutoff wavelength, either gold or beryllium coating may be selected by vertical adjustment of the entire mirror and vacuum chamber assembly to align one of the apertures and cooling channels with the synchrotron beam. The SSRL mirror has a gold coating on the entirc optical surface.

\section{Mirror Fabrication, Polishing, and Metrology}

Machining and brazing for fabrication of the misror blank was done at LBL, and electroless nicke! plating and optical polishing was done by a commercial optical company.[9] Verification of compliance with specified surface figure and roughness tolerances described above was required using standard, commercially-available instrumentation.

Surface figure (slope error) was tested with a Zygo Mark III interferometer, which provides a 
digital printout of surface height deviations from an average ideal plane on a specified spatial grid, chosen to be $3 \times 3 \mathrm{~mm}^{2}$ for these measurements. With the available equipment, only half the mirror length $(10 \mathrm{~cm})$ could be measured at one time. Maximum height deviation over the central portion of the mirror was $0.7 \lambda$ ( $\lambda=0.633$ microns). Overlapping with this central region, two additional $8 \mathrm{~cm}$ long interferograms were made at the opposite ends of the clear aperture. These yielded even smaller deviations but gave no information regarding the tilt of the reference planes among the three measurements. However, this is quite acceptable in defining slope errors since these naturally become small at the longest spatial wavelengths. These measurements were later confirmed using a Davidson Fizeau interferometer.

Surface roughness was measured with a Wyko interferometer using a $2.5 x$ objective, giving a 5 mm trace length. The main point of this process is that the use of the $5 \mathrm{~mm}$ trace Wyko profilometer plus the height measurements on a $3 \mathrm{~mm}$ grid provide coverage of the spatial wavelength spectrum with no gap in between the figure and finish measurements. This has only recently become possible using commercially-available instrumentation. On the NSLS mirror, measured roughness for spatial wavelengths between 5 microns and $5 \mathrm{~mm}$ was $7.5 \AA$, with little variation over the entire surface. At the time of this writing, polishing of the SSRL minor is in progress.

\section{Mirror Performance at NSLS}

The first mirror system was installed at NSLS on X-17T, a temporary beam line with a 5-period mini-undulator source, which was intended to provide scientific experimental opportunities while awaiting completion and installation of the soft $\mathrm{x}$-ray undulator for the $\mathrm{X}-1$ beam line. The mirror system was used for about 3 months before the beam line was disassembled and moved to its final location. Since thermal loading on the miror was only a small fraction of the design loads, it is somewhat premature to evaluate the optical performance. However, using a shearing interferometer set-up, measurements of surface figure errors due to thermal distortion were made at incident power levels of approximately $5 \mathrm{~W}$, with no measurable distortion of the optical surface, within about $\lambda / 10$ accuracy. $[10,11]$. The mechanical system performance exceeded our requirements, with excellent positioning resolution and reproducibility and UHV operation in the range of 1 nanotorr. 


\section{Potential for Future High Power Synchrotion Sources}

Although the optical and thermal loading requirements for the SSRL and NSLS beam lines have largely driven the development of this water-cooled mirror system, many aspects of the configuration were designed in anticipation of new, high power synchrotron sources on existing storage rings as well as the future 1-2 GeV Synchrotron Light Source at LBL and Advanced Photon Source at Argonne National Laborytory. Without major design modifications, the mirror system is suitable for a variety of optical systems on synchrotron radiation beam lines in which water-cooling is required in order to achieve high performance goals.

\section{Acknowledgements}

We want to thank Tony Catalano, John Lhin, Don Davis, Egon Hoyer, Hans Krapf, and John Meneghetti at LBL for their efforts and contributions during design, fabrication, assembly, and installation of the mirror systems; and Bill Lowrey and Mike Topping at CVI Laser Corp., Albuquerque, N.M. for suggestions on mirror substrate design and polishing. We are grateful to Peter Takacs for making interferometric measurements of the mirror on X-17T Beam Line and providing us with the data.

\section{Legal Notices}

This paper was prepared as an account of work sponsored by an agency of the United States Government. Neither the United States Government nor any agency thereof, nor any of their employees, makes any warranty, express or implied, or assumes any legal liability or responsibility for the accuracy, completeness, or usefulness of any information, apparatus, product, or process disclosed, or represents that its use would not infringe privately owned rights. Reference herein to any specific commercial product, process, or service by trade name, trademark, manufacturer, or otherwise, does not necessarily constitute or imply its endorsement, recommendation, or favoring by the United States Government or any agency thereof. The views and opinions of authors expressed herein do not necessarily state or reflect those of the United States Government or any agency thereof. 
This report was done with support from the Department of Energy. Any conclusions or opinions expressed in this report repr $s$ sent sulely those of the author(s) and not necessarily those of 'The Regents of the University of California, the Lawrence Berkeley Iaboratcry, or the Department of Energy. Reference to a company or product name does not imply approval or recommendation of the product by the University of Califormia or the U.S. Department of Energy to the exclusion of others that may be suitable.

\section{References}

1. R.T. Avery, "Thermal Problems on High Flux Beam Lines;" Nucl. Instr. and Meth. 222 (1984), 146-158.

2. R. DiGennaro, W.R. Edwards, and E. Hoyer, "Predicting Thermal Distortion of Synchrotron Radiation Mirrors with Finite Element Analysis," SPIE Vol. 582, Intemational Conference on Insertion Devices for Synchrotron Sources (1985) 273-280.

3. UNS Copper Alloy C15715 is a proprietary alloy ("GlidCop AL-15") by SCM Metal Products, Chemicals Division of SCM Corp. It is OFHC copper which contains $0.15 \%$ (by weight) aluminum oxide particles. Strengthening is achieved by dispersion of the particles which act as barriers to dislocation movement, according to the manufacturer. By this merhanism, high strength is achieved without significant reduction in thermal conductivity, which makes it a good candidate material for high heat flux applications. A low-oxygen version can be exposed to hydiogen at high temperatures (for fumace brazirig) withour imbrittlement.

4. H. Rarback, et al, "An Undulator Source Beamline for Soft X-Ray Imaging", Nucl. Instr. and Meth. A246 (1986), 159-162.

5. C. Jacobsen and H. Rarback, "Predictions on the Performance of the Soft X-Ray Undulator," SPIE Vol. 582, International Conference on Insertion Devices for Synchrotron Sources (1985) 201-212.

6. R. DiGennaro, "Copper Alloy C15715 Extrusion - Tensile and Metallographic Test Results", LBL Note L.BID 1138, February 1986. (Tensile test measurements confirmed the published 
mechanical properties for fuily annealed GlidCop AL-15: Ultimate Tensile Strength - 61,000 psi, $0.2 \%$ Yield Strength - 49,000 psi, and $0.01 \%$ Yield Strength - 35,000 psi.)

7. Brazing of the alumina dispersion-strengthened copper is difficult due to its fine grain size which tends to promote rapid intergranular diffusion of the braze material, thus depleting and weakening the brazed joint. To reduce this effect, the manufacturer recommends avoiding the use of silver-based braze alloys and minimizing the time at which the parts are kept above the liquidus temperature ("flow-point"). Successful brazing of the mirror assembly was done by copper-plating the joints prior to brazing, which reduced intergranular diffusion of the braze material. "Palcusil 25 " alloy (54\% Au, 21\% Cu, 25\% Pd) was used to braze the water-channel inserts to the copperplated substrate, and was furnace brazed in an argon atmosphere. For other applications in which stairless steel tubing was brazed directly to dispersion-strengthened copper (without plating), successful results were achieved using a foil braze alloy which contained $50 \% \mathrm{Au}, 50 \% \mathrm{Cu}$. In all applications, the low-oxygen grade of GlidCop AL-15 was used.

8. G. Lee, "Rádiation Resistance of Elastomers," IEEE Trans. on Nuclear Science, Vol. NS-32, No. 5 (1985), 3806-3808.

9. Mirror polishing was done by CVI Laser Corp., Albuquerque, New Mexico.

10. Private communication with Peter Taicacs at NSLS.

11. Shi-Nan Qian, H. Rarback, D. Shu, and P. Z. Takacs, "In-situ Shearing Interferometry of National Synchrotron Light Source Mirrors," SPIE Electro-Optic Imagining Systerius and Devices Symposium, Los Angeles, Jan. 11-16, 1987 


\section{Figure Captions}

Figure 1. Two similar water-cooled, plane-deflection mirror systems are being built. One is to be installed on the X-1 Beam Line at NSLS (Fig. 1a), and the other is to be installed on a new VUV Branch Line of Beam Line VI at SSRL (Fig. 1b).

Figure 1a. NSLS X-1 Bearn Line. Source size : $2 \sigma_{\mathrm{H}}=1 \mathrm{~mm}, 2 \sigma_{\mathrm{V}}=0.2 \mathrm{~mm}$.

Figure 1b. LBL/Exxon/SSRL Beam Line VI - VUV Branch Line. Source size: $2 \sigma_{\mathrm{H}}=8 \mathrm{~mm}$, $2 \sigma_{\mathrm{V}}=0.6 \mathrm{~mm}$.

Figure 2. Finite element calculations were done to estimate mirror temperatures, stresses, and distortion due to themal loading. Fig. 2a describes the 2-dimensional mesh model utilizing symmety to model $1 / 2$ of the mirror cross-section and thermal boundary conditions. The 2 -d model was used to optimize the cooling channel geometry for minimum thermal distortion at acceptable stress levels. The 3-dimensional model shown in Fig. $2 b$, based on $1 / 4$ symmetry, was used for thermal stress calculations to achieve an accurate representation of the true stress state without using 2-d approximations.

Figure 2a. $2 \mathrm{~d}$ finite element model for thermal distortion analysis and design optimization.

Figure 2b. 3-d finite element model for thermal stress analysis.

Figure 3. Finite element analysis results with 2-d model. Fig. 3a shows temperature contours - at maximum power, peak surface temperature will be less than $70^{\circ} \mathrm{C}$, with $\Delta \mathrm{T}$ of about $20^{\circ} \mathrm{C}$ across the $3 \mathrm{~mm}$ wall thickness to the cooling channel, In Fig. 3b, the undistorted mirror mesh model (dashed lines) is overlayed with the distoned shape (solid lines), showing the distorions magnified by a scale factor of 400 . Maximum sagittal slope etror occurs near the edge of the illuminated region, less than 60 arcsec at maximum power conditions on Beam Line VI, and less than 8 arcsec on $X-1$. 
Figure 3a. Isothermal contour lines in the mirror substrate near the illuminated region and cooling channel.

Figure 3b. Thermal distortion (magnified, shown with solid lines) overlayed on the undistorted shape (dashed lines).

Figure 4. The mirror substrate is a furnace-brazed assembly with machined internal water-cooling channels, shown in Fig. $4 \mathrm{a}$ with the inserts, before brazing. Both supply and return water channels are close to the optical surface for optimum cooling, and the single water circuit also provides coolant for an upstream mask which is mounted directly to the mirror assembly. The box-like mirror back shown in Fig. $4 \mathrm{~b}$ is brazed to the front substrate to provide an air-jacket around all water connections and yent to atmosphere, and also isolates mounting and clamping forces to prevent distortion of the optical surface.

Figure 4a. Mirror substrate machined cooling channels and inserts for brazing.

Figure $4 \mathbf{b}$. Brazed internal assembly with a single water circuit for cooling both the substrate and upstream mask.

Figure 5. Mirror mounting and mechanism which provides positioning ad ustments for three degrees of freedom. Flexural pivots align with the vertical $Y-Y$ axis for yaw positioning. For roll adjustments, a linkage assembly using flexural hinges has a center of rotation which coincides with the longitudinal $\mathrm{Z}-\mathrm{Z}$ axis at the optical surface. Water tubing and air vents pass through a joystick positioning tube. The entire in-vacuum assembly may be moved horizontally along the $\mathrm{X}-\mathrm{X}$ axis for translation in or out of the synchrotron beam.

Figure 6. Flange-mounted mirror system before polishing. At installation, a water-cooled upstream mask (not shown) is mounted to the mini-conflat flanges seen in the foreground, with internal o-ring fittings for water connections.

Figure 7. Flange-mounted mirror system assembly with linear slides and stepper motors for positioning adjustments. 
Figure 8. Complete mirror assembly and water-cooled mask. Photograph was taken after the mirror was removed from the temporary X-17T mini-undulator beam line at NSLS. 

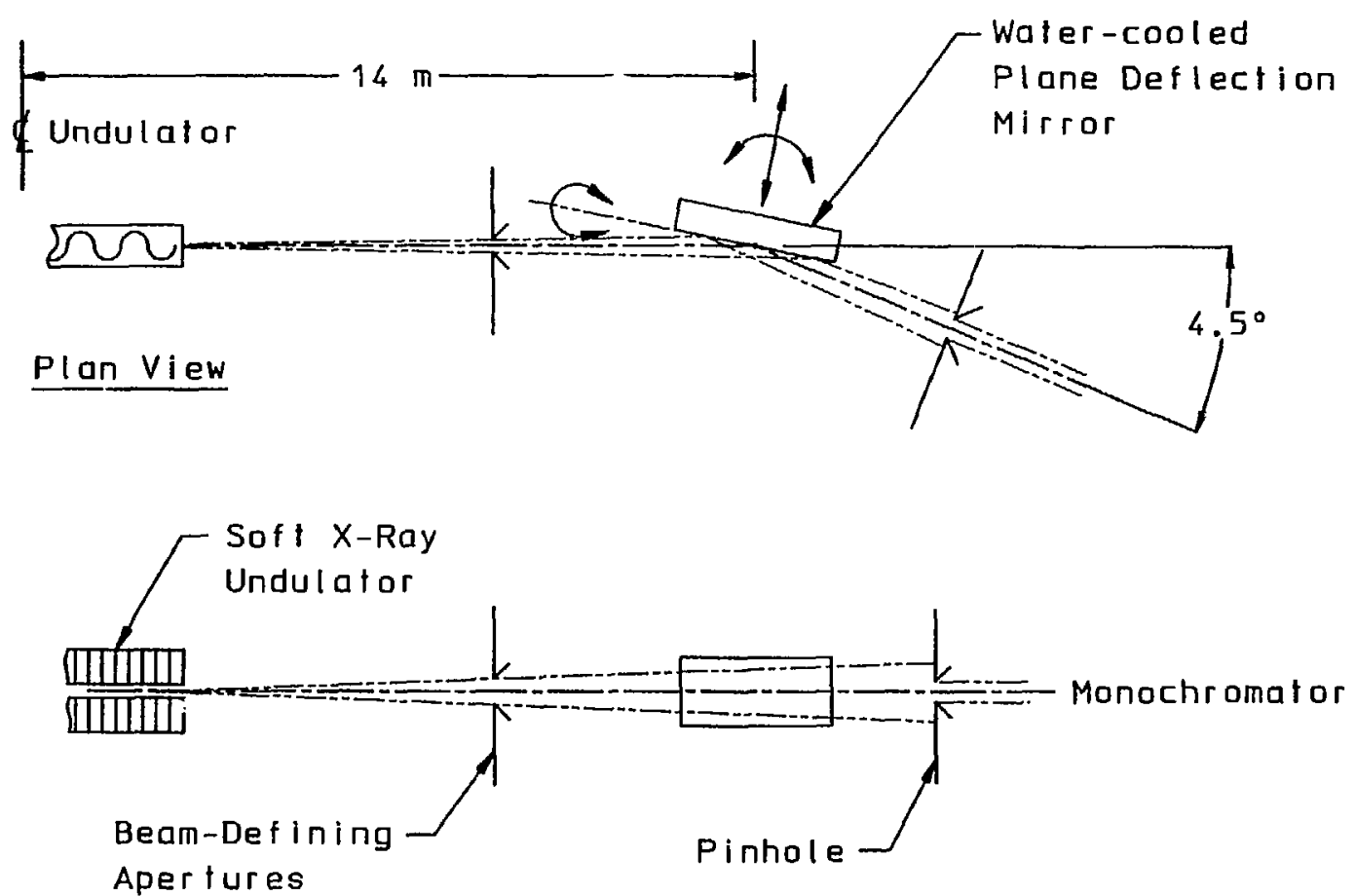

Elevation 


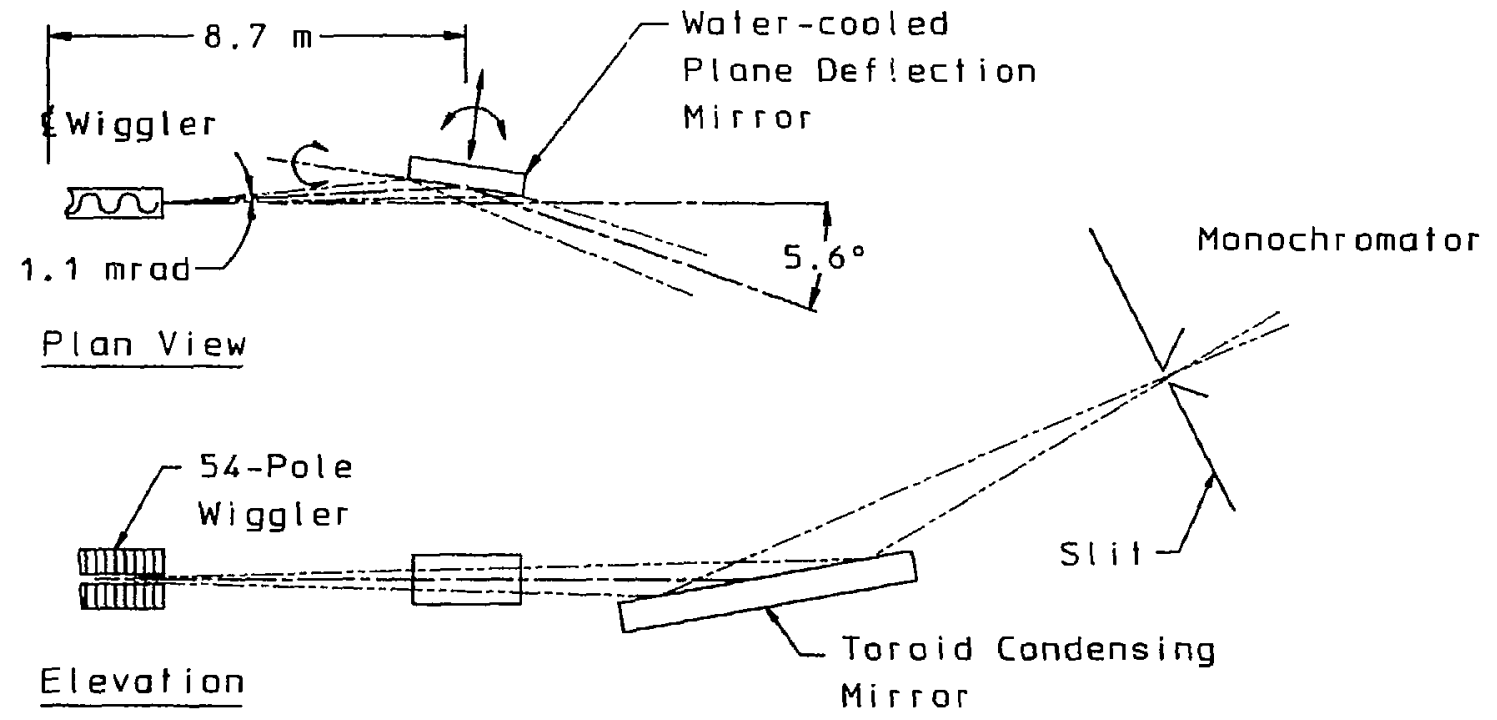

\section{LBL / EXXON/SSRL BEAM LINE VI VUV BRANCH LINE}




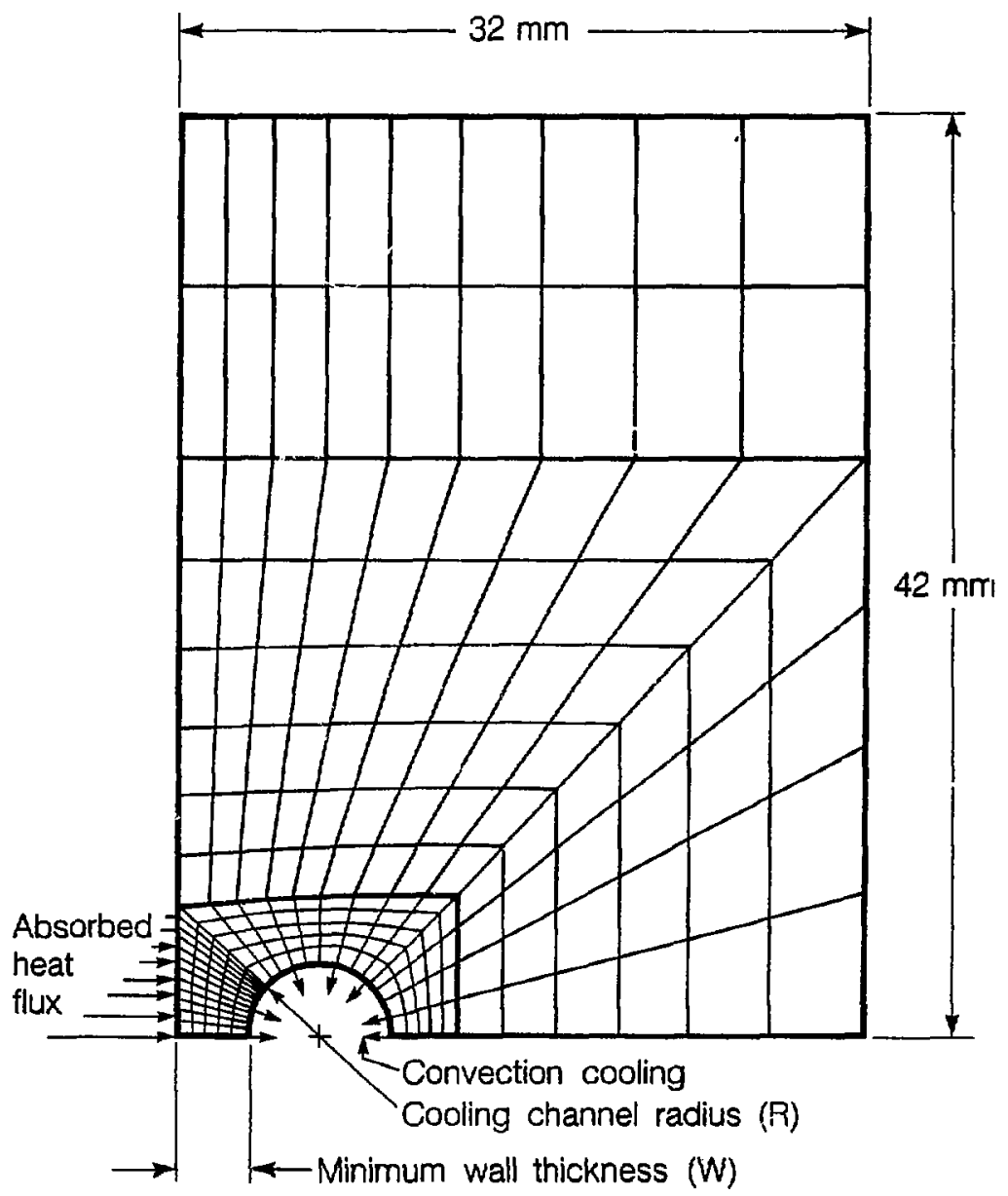




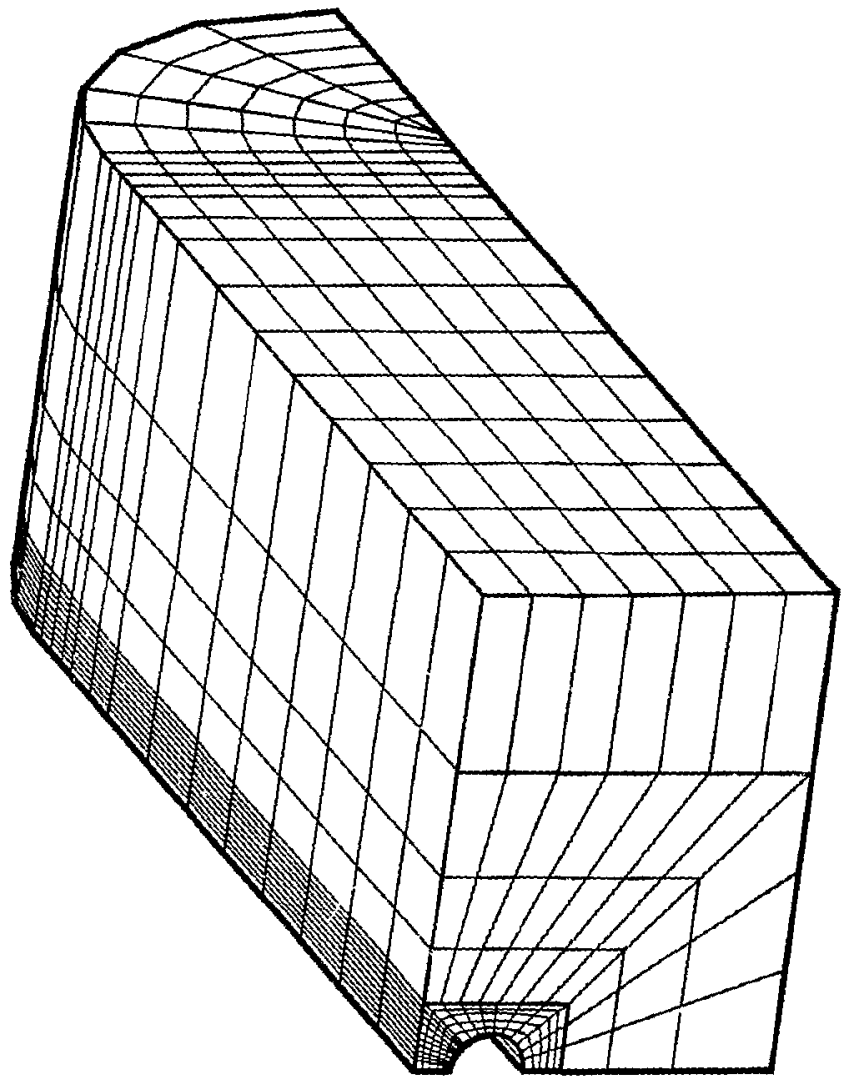

Fig. 2b

XEL 8510.11716 


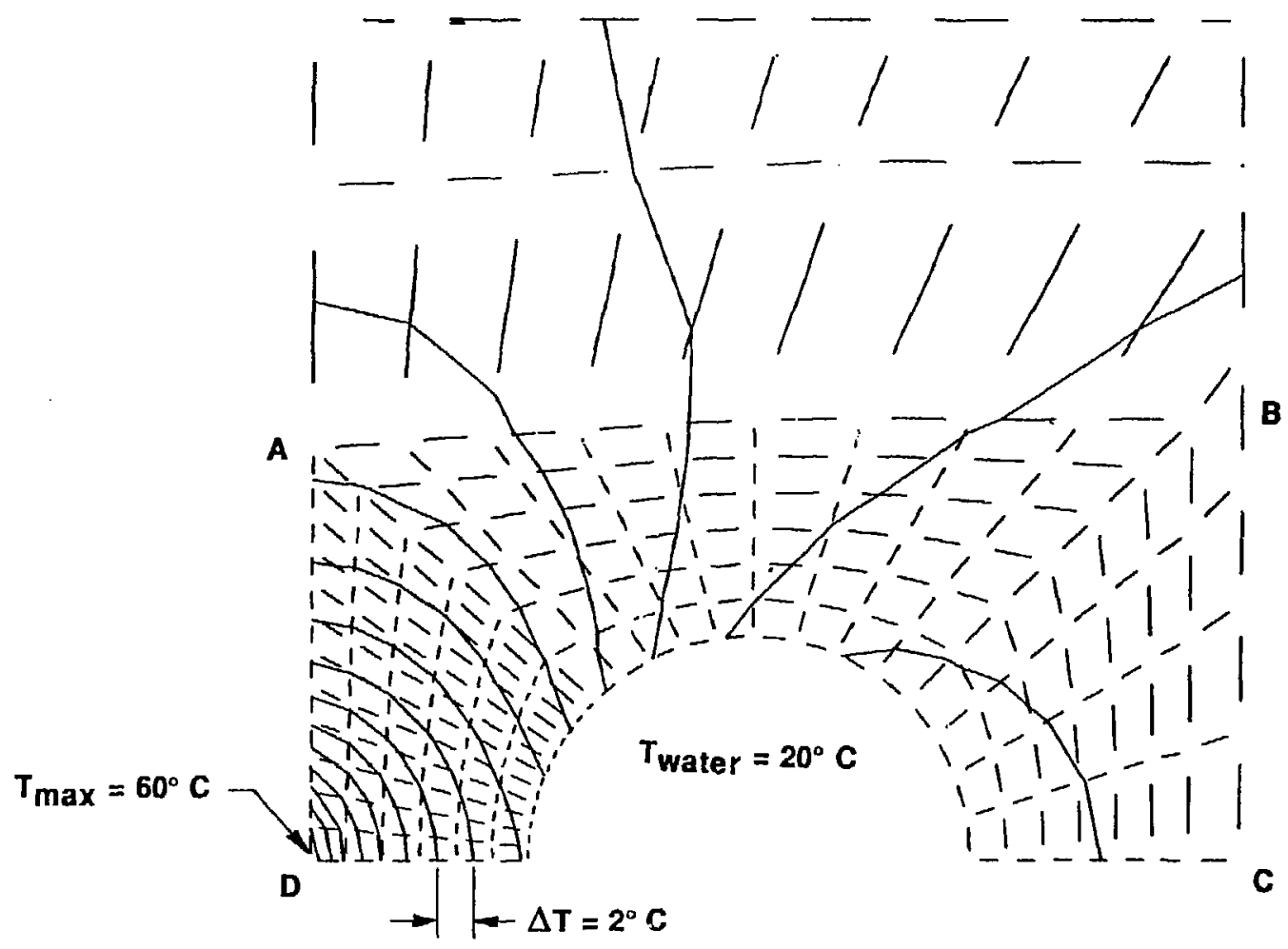

Figure 3a. Isothermal contour lines in the mirror substrate near the illuminated region and cooling channel. 


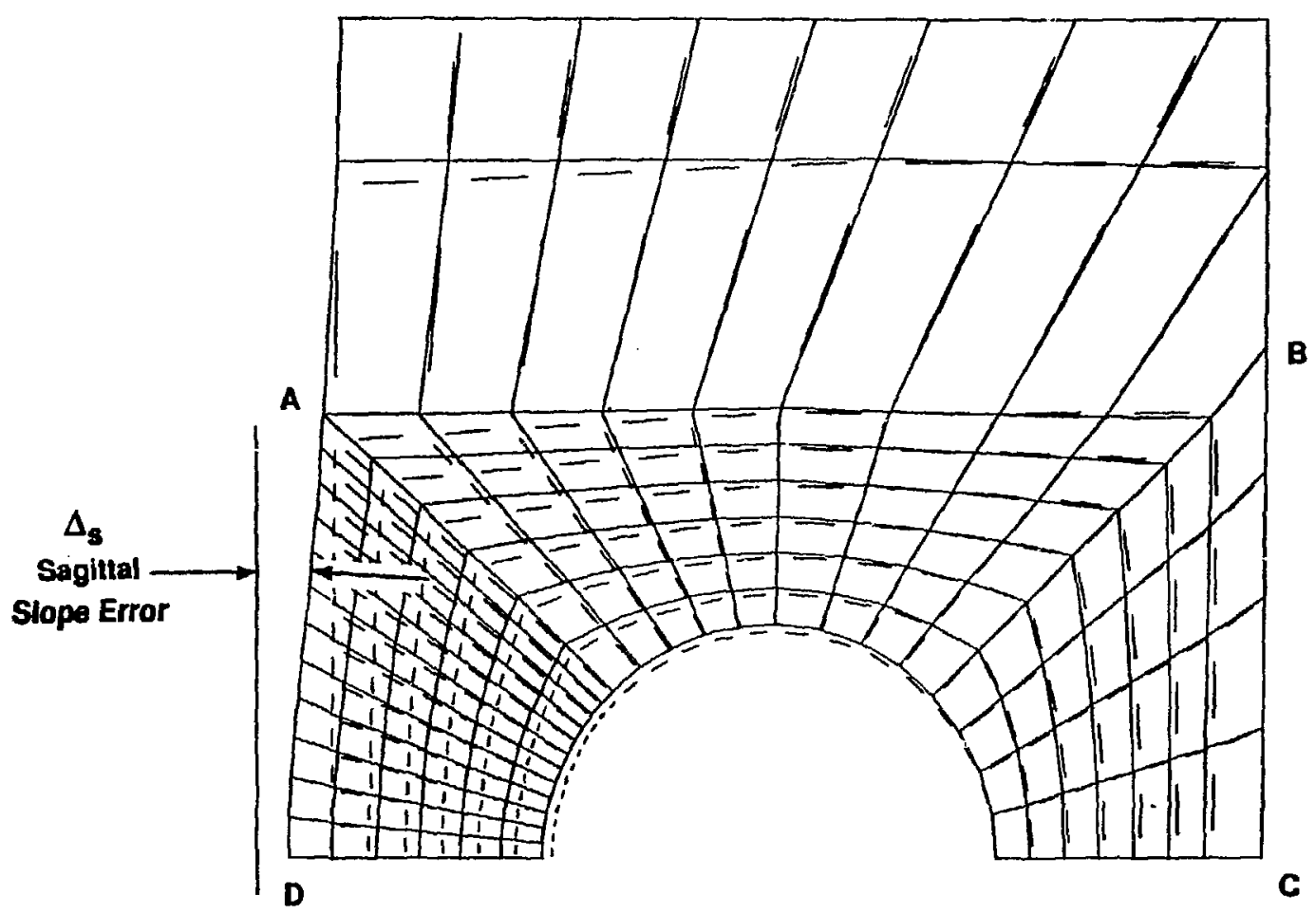

Figure 3b. Thermal distortion (magnified, shown with solid lines) overlayed with the undistorted shape (dashed lines). 


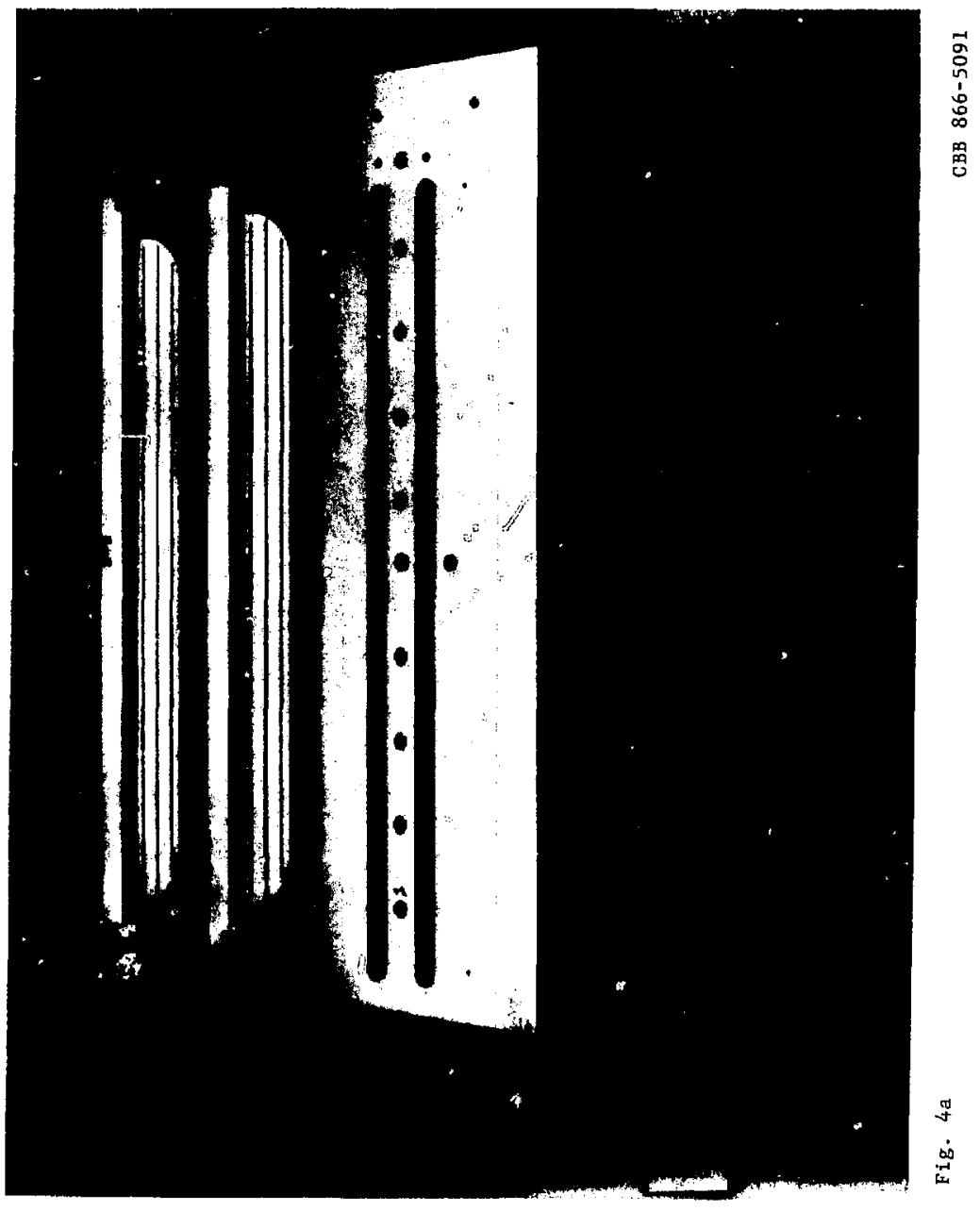




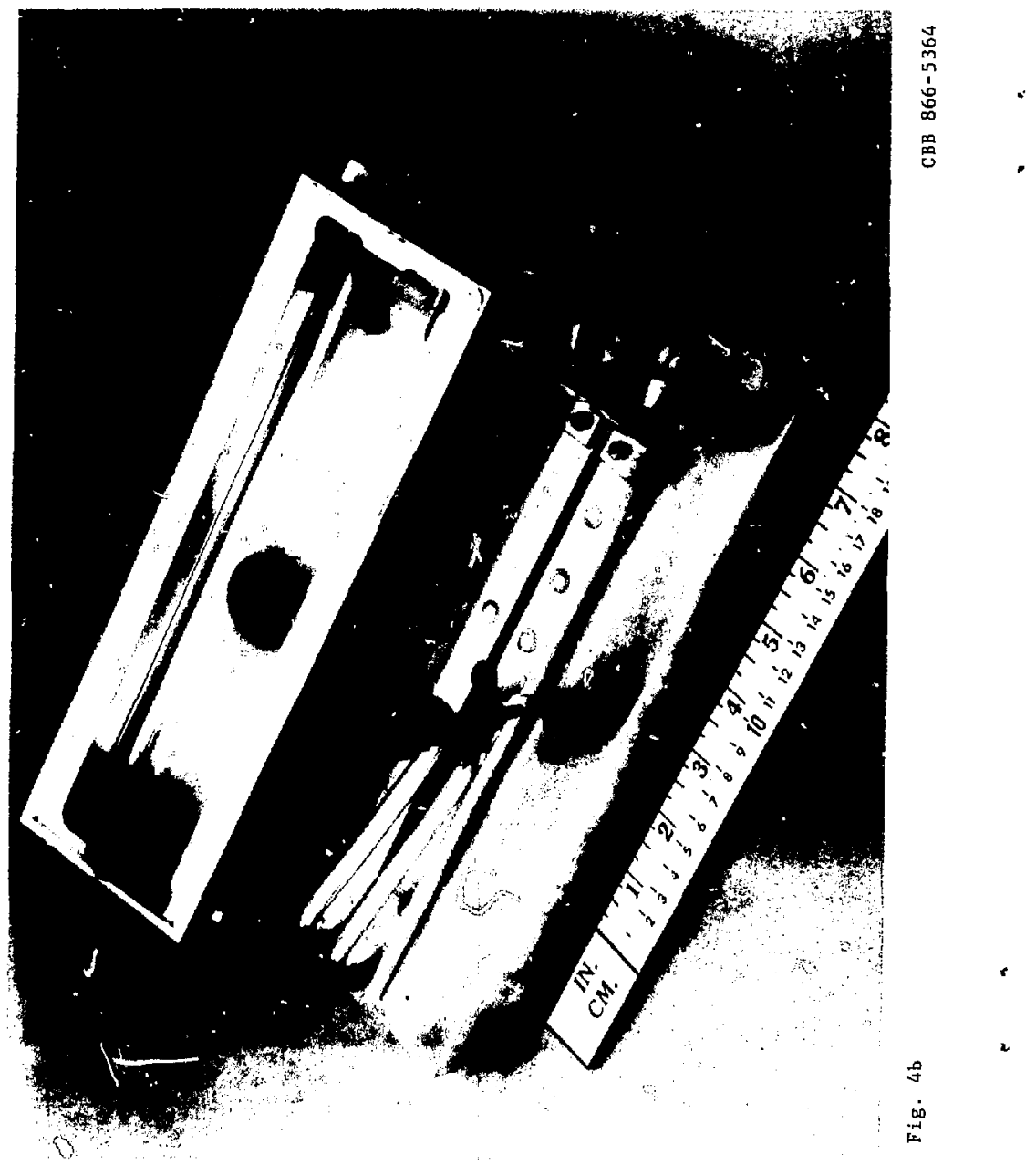




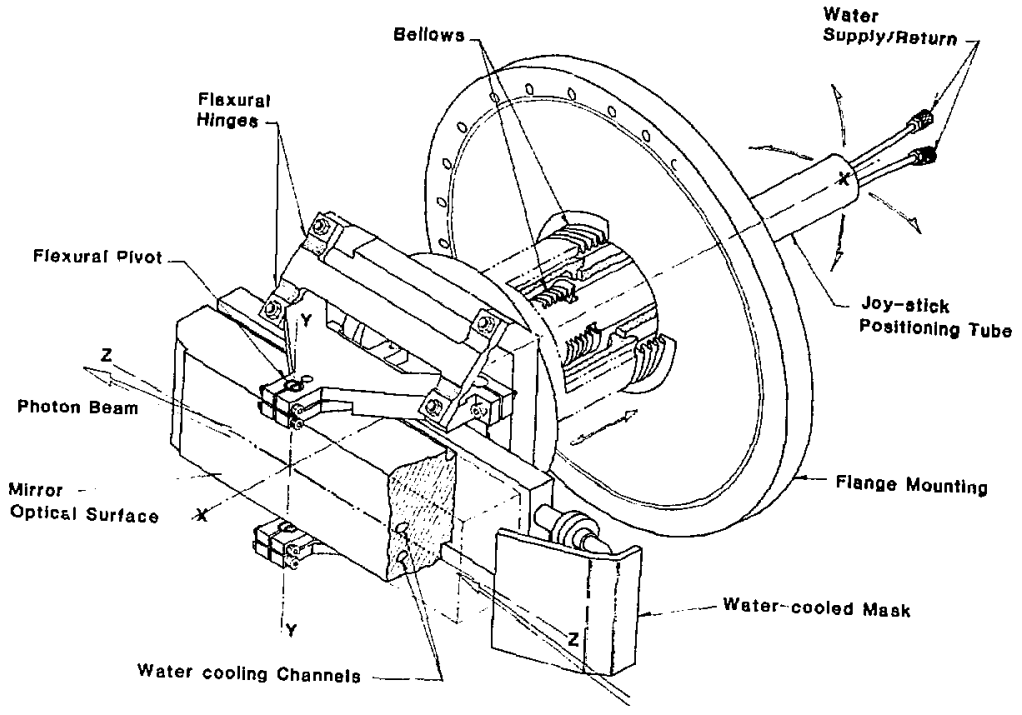

XBL 865-1979 


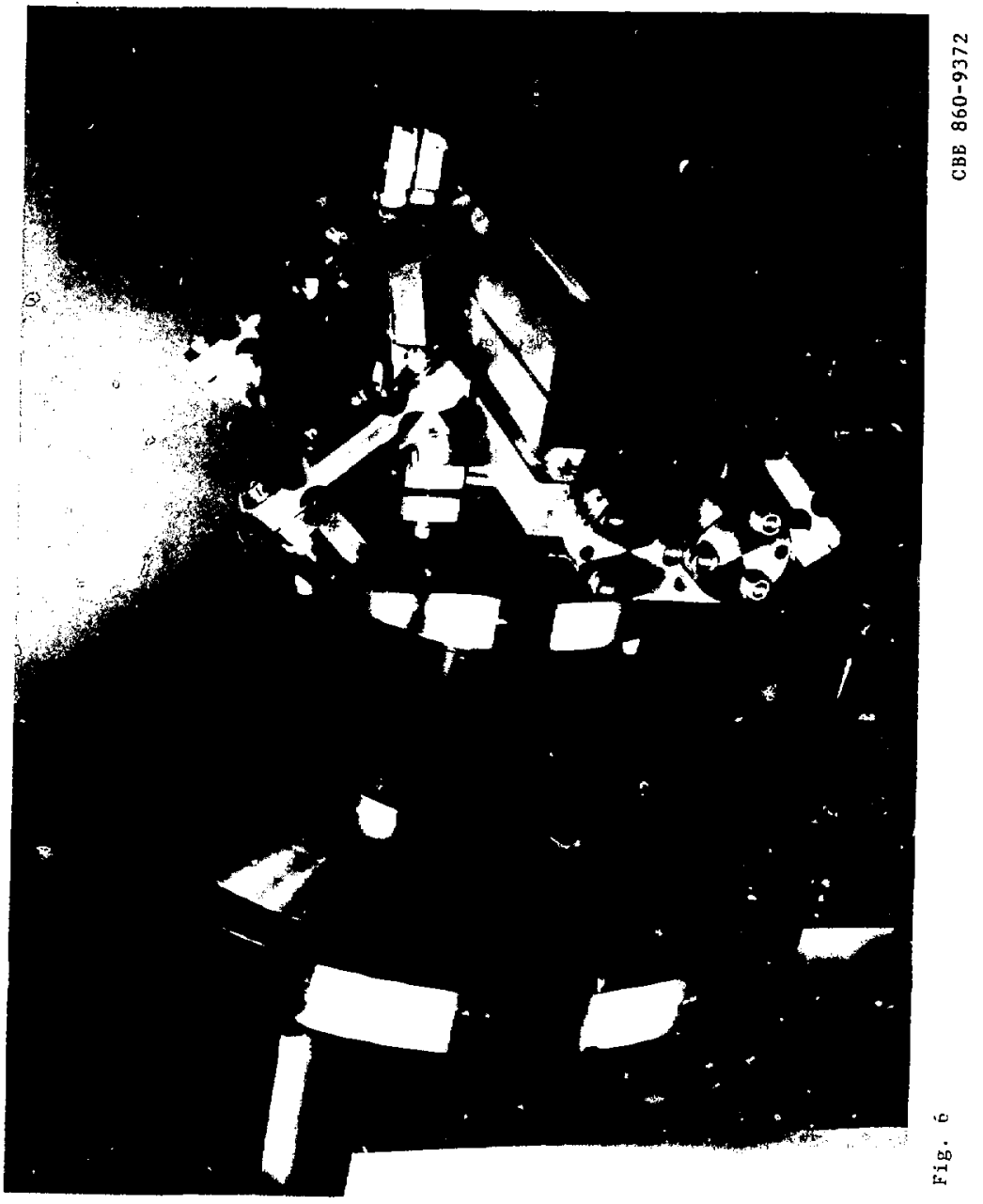

4 


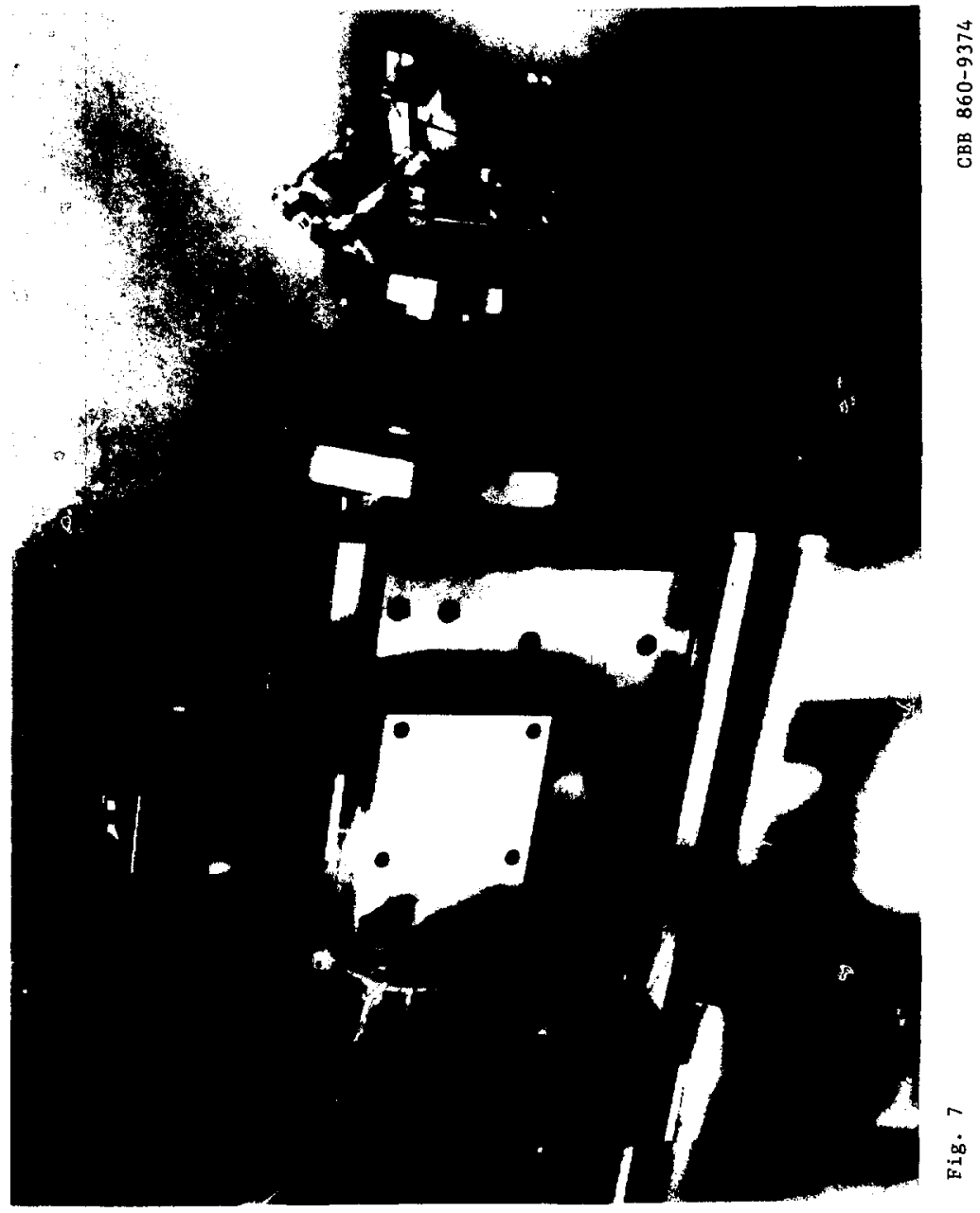




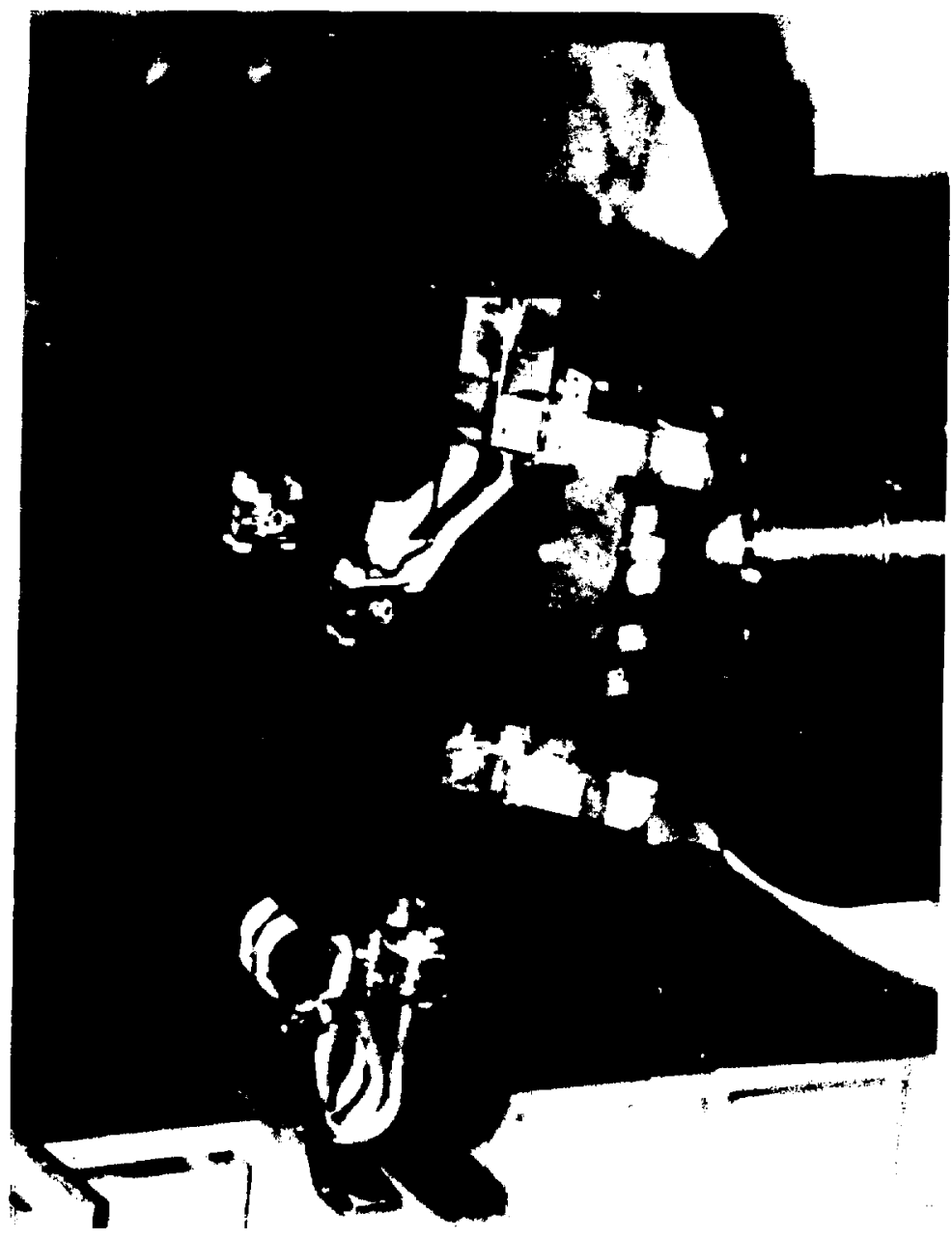

9
0
0
0
$\vdots$
0
0
0
0
0

$$
\text { • }
$$

